\title{
Reproducción del camarón rosado Farfantepenaeus notialis (Decapoda: Penaeidae) en el Caribe colombiano
}

\author{
Jorge Paramo ${ }^{1}$, Daniel Pérez ${ }^{1} \&$ Matthias Wolff ${ }^{2}$ \\ 1. Universidad del Magdalena, Grupo de Investigación Ciencia y Tecnología Pesquera Tropical (CITEPT). Cra. 32 No. \\ 22-08 Avenida del Ferrocarril, Santa Marta; jparamo@unimagdalena.edu.co, danieldm90@gmail.com \\ 2. Universität Bremen, Leibniz Zentrum für Marine Tropenökologie (ZMT). Fahrenheit Strasse 6, Bremen, Alemania; \\ matthias.wolff@zmt-bremen.de
}

Recibido 21-VIII-2013. Corregido 15-XII-2013. Aceptado 22-I-2014.

\begin{abstract}
Reproduction of the pink shrimp Farfantepenaeus notialis (Decapoda: Penaeidae) in the Colombian Caribbean. The shallow water pink shrimp (Farfantepenaeus notialis) is among the socioeconomically most important resources of the Caribbean. The lack of biological and fishery information is of great concern for the fisheries management authorities. The presented study therefore aimed at the investigation of the reproductive cycle, the size composition and the size at first maturity of this species as a basis for the ordination and management of this resource. The study was conducted from June 2012 to May 2013 off the coast of the Caribbean Sea of Colombia. A total of 5356 individuals were collected, identified, classified and preserved for their subsequent analysis in the laboratory. Size, weight, sex and gonad stage were recorded for each specimen. Significant differences were found in sex ratio in all months sampled with a clear predominance of females. Mature females were found year-around, but two reproductive peaks were identified during the periods OctoberDecember and April-June. The mean catch total length size (MCS) for females and males was $148.00 \mathrm{~mm}$ and $122.54 \mathrm{~mm}$, respectively. The mean size at maturity $\left(\mathrm{LT}_{50 \%}\right)$ was $129.34 \mathrm{~mm}$ for females and $97.77 \mathrm{~mm}$ for males. MCS was always above $\mathrm{LT}_{50 \%}$ for both sexes. Considering the large reduction in fishing effort in the Colombian Caribbean Sea over the last years, we could expect that the shrimp population is in a rebuilding process or perhaps it may be already restored. Rev. Biol. Trop. 62 (2): 513-521. Epub 2014 June 01.
\end{abstract}

Key words: shrimps, Farfantepenaeus notialis, reproduction, maturity, Colombian Caribbean.

En el Caribe colombiano la pesquería de camarón de aguas someras (CAS) tiene como objetivo principal la captura del camarón rosado (Farfantepenaeus notialis, Pérez-Farfante, 1967), aunque también están presentes otras especies de camarones con menores capturas tales como Farfantepenaeus brasiliensis (Latreille, 1817), Farfantepenaeus subtilis (Pérez-Farfante, 1967) y Litopenaeus schmitii (Burkenroad, 1936) (Paramo \& Saint-Paul, 2010). La pesquería de camarón de aguas someras es de gran importancia socio-económica en la región Caribe. Así como en la mayoría de las pesquerías tropicales de camarón alrededor de mundo que están completamente explotadas (Guillet, 2008), este es un típico caso de una pesquería colapsada por falta de control (Paramo \& Saint-Paul, 2010). No obstante, debido a la sobreexplotación, falta de información biológica, pesquera, seguimiento y control, es necesario generar conocimiento biológicopesquero sobre el recurso. De tal manera, que los tomadores de decisiones y/o entidades competentes puedan elaborar y tomar medidas de manejo para su aprovechamiento racional y sostenible, considerando el enfoque precautorio de acuerdo con el código de conducta para la pesca responsable (FAO, 1995). Este enfoque considera información básica sobre la biología reproductiva de la población de camarón tales como estadios reproductivos, talla media de madurez, época reproductiva y zonas 
donde se reproducen. La sobreexplotación del recurso debido a la alta presión ejercida sobre un amplio ámbito de tallas, como consecuencia de la falta de medidas de manejo (Paramo \& Saint-Paul, 2010), ha tenido como consecuencia la disminución de la flota pesquera industrial en las últimas décadas, que se viene presentando en muchos mares tropicales a nivel mundial (García, 1989). Situación que ha sido documentada por el Instituto Colombiano de Desarrollo Rural (INCODER) y la Autoridad Nacional de Acuicultura y Pesca (AUNAP) en sus estadísticas pesqueras en el Caribe colombiano (Barreto \& Borda, 2008; Paramo \& Saint-Paul, 2010). Por ello, es de gran relevancia tener información precisa sobre del estado actual del stock y de su biología reproductiva (Desantis et al., 2001; Belcari, Viva, Mori \& De Ranieri. 2003; Carlucci D’Onghia, Sion, Maiorano \& Tursi, 2006; Kapiris \& Thessalou-Legaki, 2006, 2009; Echeverría-Sáenz \& Wehrtmann, 2011). El camarón rosado ( $F$. notialis) pertenece a la familia Penaeidae, es de gran importancia comercial para la pesca industrial en el Caribe colombiano (Paramo \& Saint-Paul, 2010). F. notialis habita en fondos blandos desde los 3 a los $100 \mathrm{~m}$ y raramente llega hasta los $700 \mathrm{~m}$, pero presenta sus mayores abundancias en aguas someras entre los 10 a $75 \mathrm{~m}$ de profundidad (Fischer, Bianchi \& Scott, 1981). F. notialis se distribuye a lo largo de la costa del mar Caribe, la costa de América del Sur hasta Brasil, y también se encuentra en la costa de África Occidental desde Mauritania hasta Angola (Tavares, 2002). El ciclo de vida de los camarones penaeidos es complejo (Dall, Hill, Rothlisberg \& Staples, 1990), involucrando el desove de los adultos fuera de la costa, y la migración de las larvas hacia las regiones de crianza, que incluyen lagunas costeras, manglares y estuarios (García \& Le Reste, 1986). Las zonas de crianza proporcionan alimento (Lee, 1999; Loneragan \& Bunn, 1999) y refugio contra los predadores (Minello \& Zimmerman, 1991). La migración comprende una fase estuarina, cuando la postlarva entra a las desembocaduras de los ríos, donde se mantienen hasta la etapa juvenil y luego migran hacia el mar como sub-adultos, el cual es un período caracterizado por el rápido crecimiento y migración continua (Dall et al., 1990). El camarón de aguas someras $F$. notialis pertenece a las especies que desovan frente a la costa, a profundidades que varían aproximadamente de 10 a $80 \mathrm{~m}$. Generalmente las especies de la familia Penaeidae alcanzan su madurez sexual antes de haber cumplido un año de edad (Fischer et al., 1995). Por lo tanto, se requiere investigación biológica para conocer el ciclo reproductivo y los factores que afectan el éxito en el desove ya que el conocimiento respectivo es de alta prioridad para el manejo pesquero. Las regulaciones tales como vedas durante épocas de reproducción y el reclutamiento de los crustáceos, dependen del conocimiento de la biología reproductiva (Lizárraga-Cubedo, Pierce \& Santos, 2008). Por esta razón, el presente estudio tiene como objetivo conocer la época reproductiva, la estructura de tallas y la talla media de madurez del camarón de aguas someras (Farfantepenaeus notialis) como insumo para la ordenación y manejo pesquero en el Caribe colombiano.

\section{MATERIALES Y MÉTODOS}

Muestreo a bordo del barco camaronero: Las muestras fueron recolectadas en el barco camaronero Adriatic, entre junio 2012 y junio 2013 en los sitios donde opera normalmente la flota camaronera en el Caribe colombiano (Fig. 1). Se realizaron en total 141 estaciones de muestreo, usando una red de arrastre camaronera de ancho $12.8 \mathrm{~m}$ y largo $10.7 \mathrm{~m}$, con tamaño de malla al final del copo de $44.5 \mathrm{~mm}$ de nudo a nudo. El muestreo se hizo de acuerdo a la reglamentación vigente sobre dispositivo excluidor de tortugas (DET). Para la evaluación de los fondos arrastrables se utilizó una ecosonda FURUNO FCV 1150. La duración aproximada de cada arrastre comercial fue de cuatro horas, en los cuales se tomaron datos de hora inicial y final, posición inicial y final por medio de un GPS marca Garmin GPSmap76CSx. De cada lance se tomó una muestra de aproximadamente dos kilos y medio, unos 


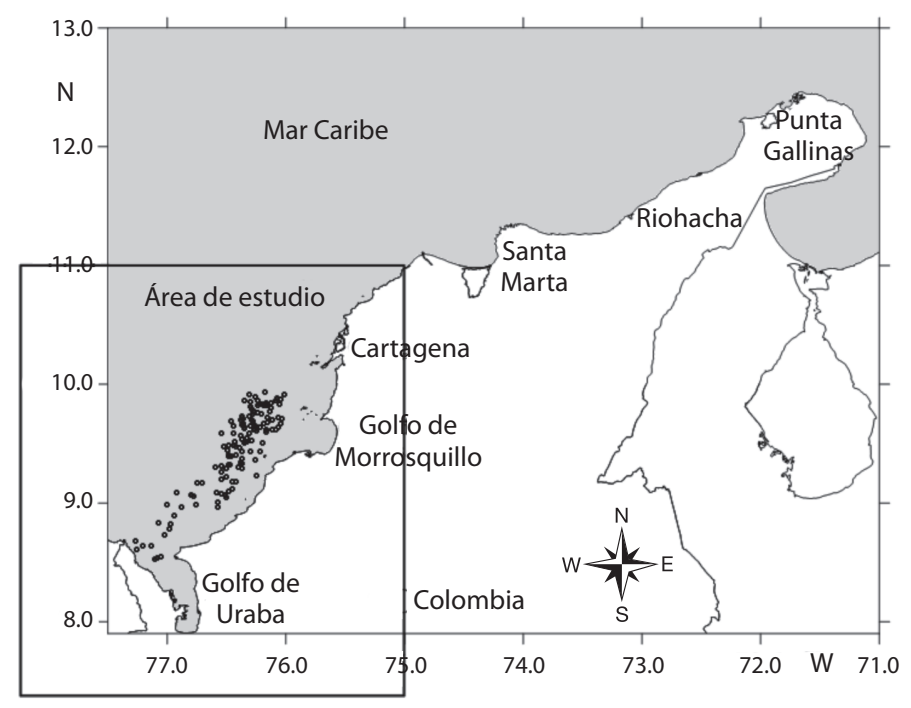

Fig. 1. Área de estudio y estaciones de muestreo.

Fig. 1. Study area and sampling stations.

50 individuos. Los individuos recolectados, fueron separados, identificados, conservados con bisulfito de sodio y guardados en bolsas debidamente rotuladas para su análisis en laboratorio del Centro de Desarrollo Pesquero y Acuicola en Taganga de la Universidad del Magdalena. En cada individuo se registró la longitud total (LT) desde la punta del rostro hasta la punta del telson con un calibrador digital de 12" UPM (módulo de presión universal) con precisión de $0.01 \mathrm{~mm}$, y el peso total con una balanza analítica marca Ohaus Pioneer con una precisión de $0.1 \mathrm{mg}$.

Determinación macroscópica de los estadios de madurez gonadal: Se determinaron visualmente cinco estadios de madurez macroscópicos para las hembras y machos: I) inmaduros, ovarios translúcidos; II) en desarrollo, ovarios opacos; III) casi maduros, ovarios de color amarillo naranja; IV) maduros, ovarios grandes color oliva, algunas veces café; y V) desovados, ovarios vacíos. Para la estimación de la talla media de madurez se consideraron los estadios I y II como inmaduros y los estadios III, IV y V como maduros (Paramo \& Saint-Paul, 2010).
Se determinó la proporción sexual, el estadio de madurez gonadal por sexos. Se realizó una prueba de Chi-cuadrado para establecer significancia estadística de diferencias entre el número de hembras y machos en las muestras mensuales. Se evaluó las diferencias en la distribución de frecuencias de las tallas entre hembras y machos usando la prueba no paramétrica Kolmogorov-Smirnov (Gotelli \& Ellison, 2004). Se realizó una prueba de comparaciones múltiples de Kruskal-Wallis para determinar el nivel de significancia estadística entre las diferencias de la talla media de captura (TMC) mensual en hembras y machos de $F$. notialis. Se calculó para ambos sexos por muestra mensual la proporción de individuos maduros e inmaduros de $F$. notialis. La talla media de madurez sexual $\left(\mathrm{LT}_{50 \%}\right)$ fue modelada ajustando la función logística de la proporción de individuos maduros con $5 \mathrm{~mm}$ de intervalo de clase de talla de LT (Paramo \& Saint-Paul, 2010), mediante una regresión no lineal. Se realizó una prueba de Chi-cuadrado para evaluar el nivel de significancia estadística en las diferencias entre la talla media de captura mensual con la talla media de madurez en hembras y machos, respectivamente. 


\section{RESULTADOS}

Proporción de sexos: Se analizó un total de 5199 individuos, $56.4 \%$ hembras y $43.6 \%$ machos (Fig. 2). En todos los meses predominaron las hembras, con mayor porcentaje en septiembre y octubre, y el más bajo en noviembre (Fig. 2). Existen diferencias significativas entre la abundancia de hembras y machos en todos los meses muestreados $\left(\mathrm{Chi}^{2}=591.63\right.$; $\mathrm{p}=0.000$ ).

Estructura de tallas: La estructura de tallas en todo el periodo muestra un intervalo para las hembras desde 69.7 hasta $198.6 \mathrm{~mm}$ de LT, con una media de $148.0 \mathrm{~mm}$ de LT $(\mathrm{CV}=12.1 \%)$ (Fig. 3A). Para los machos se obtuvo un intervalo desde 74.0 hasta $181.8 \mathrm{~mm}$ de LT, con una media de $122.5 \mathrm{~mm}$ de LT (CV=10.4\%) (Fig. 3B). En todos los meses las hembras fueron significativamente de mayor talla que los machos ( $p=0.000)$. La TMC mensual de las hembras fue mayor entre junio y diciembre 2012 ( 150mm de LT) y disminuyó en enero, marzo y mayo $(\sim 135 \mathrm{~mm}$ de LT), mostrando diferencias significativas entre estos dos periodos (Fig. 3A) $(\mathrm{p}=0.000)$. La TMC de los machos aumentó desde junio hasta septiembre 2012 ( 127mm de LT) y disminuyó desde enero hasta marzo $2013(\sim 115 \mathrm{~mm}$ de LT), mostrando diferencias significativas entre los meses de muestreo ( $\mathrm{p}=0.000)$ (Fig. 3B). Los individuos fueron capturados entre 15.48 y $66.60 \mathrm{~m}$ de profundidad (profundidad media de $46.19 \mathrm{~m} \pm 11.87)$.

Porcentaje mensual de estadios de madurez: En junio $81.5 \%$ de las hembras estuvieron maduras, mientras que en septiembre el porcentaje disminuyó a $56.6 \%$ y en diciembre aumentó hasta $76.1 \%$. Posteriormente entre enero y marzo 2013 el porcentaje de hembras maduras disminuyó, para luego aumentar entre abril (80.1\%) y mayo (71.3\%) de 2013 (Fig. 4A). Los machos presentaron un $94.7 \%$ de individuos maduros en el mes de junio y el alto porcentaje se mantuvo hasta diciembre de 2012. Sin embargo, entre enero y marzo 2013 el porcentaje de individuos maduros disminuyó con valores entre $66.3 \%$ y $63.6 \%$, para luego aumentar en abril $(85.3 \%)$ y mayo $(76.6 \%)$ de 2013 (Fig. 4B).

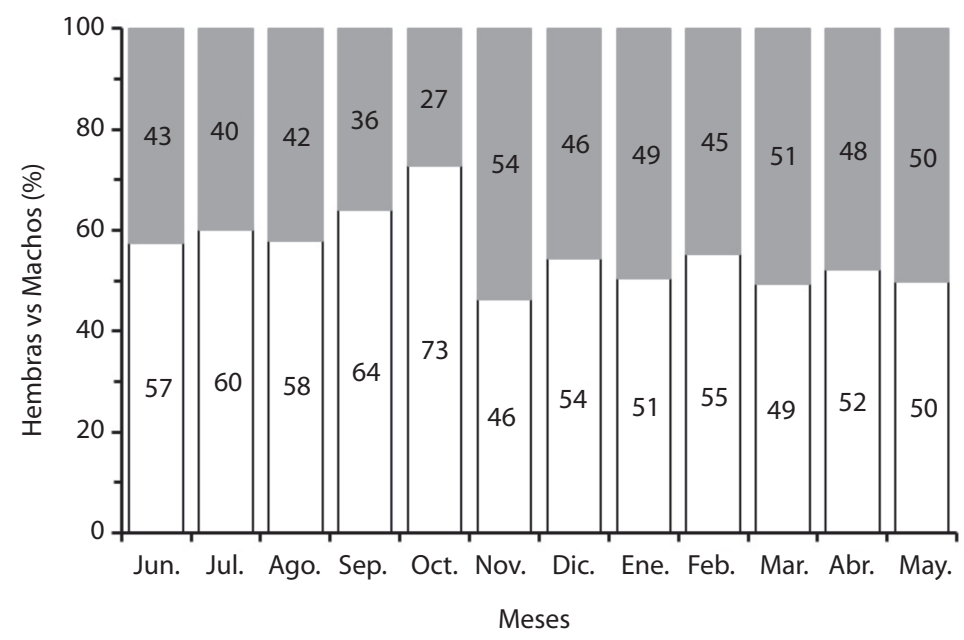

Fig. 2. Porcentaje de hembras (blanco) y machos (gris) del camarón rosado Farfantepenaeus notialis, obtenidas durante los muestreos entre junio 2012 y mayo 2013.

Fig. 2. Percentage of female (white) and male (grey) of the pink shrimp Farfantepenaeus notialis, obtained during the samplings between June of 2012 and May of 2013. 

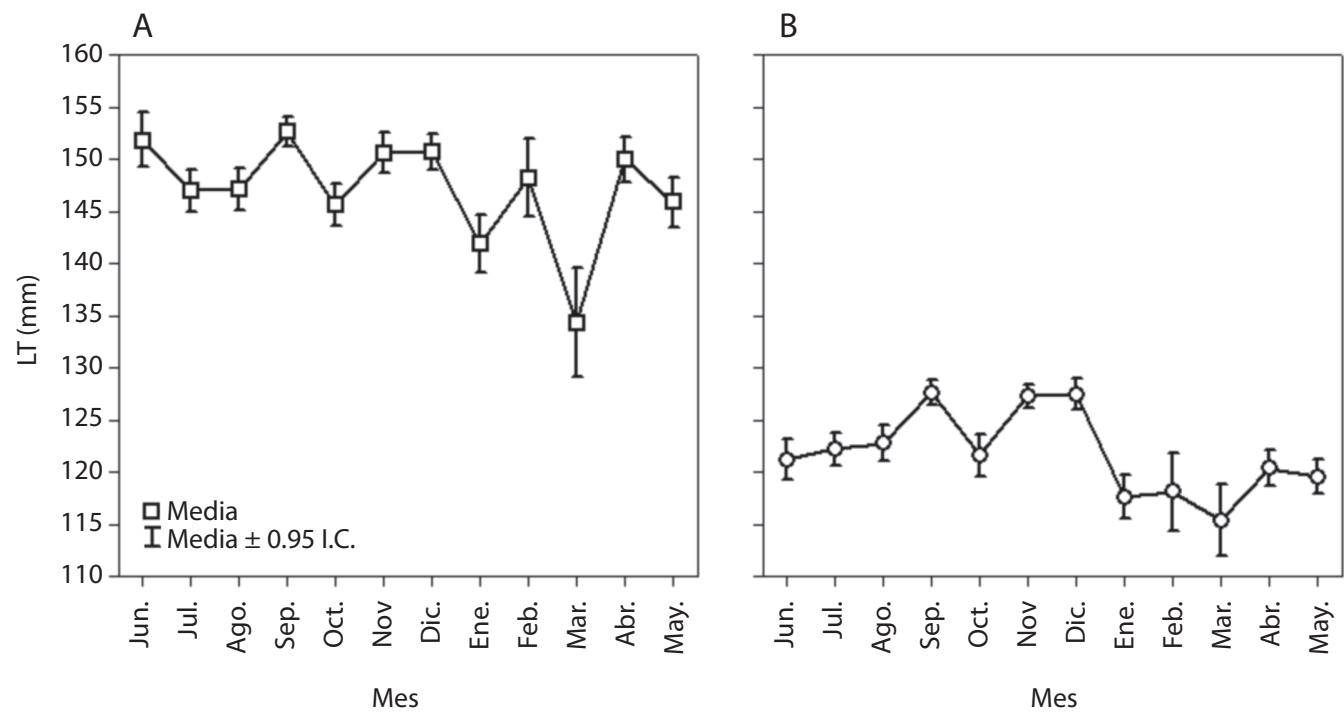

Fig. 3. Talla media de captura, longitud total (LT) en $\mathrm{mm}$ para (A) hembras y (B) machos de los individuos de Farfantepenaeus notialis, obtenidos durante los muestreos mensuales desde junio 2012 hasta mayo 2013.

Fig. 3. Mean size of catch, total length (TL) in $\mathrm{mm}$ to (A) females and (B) males of individuals of Farfantepenaeus notialis, obtained during the monthly samplings from June of 2012 to May of 2013.
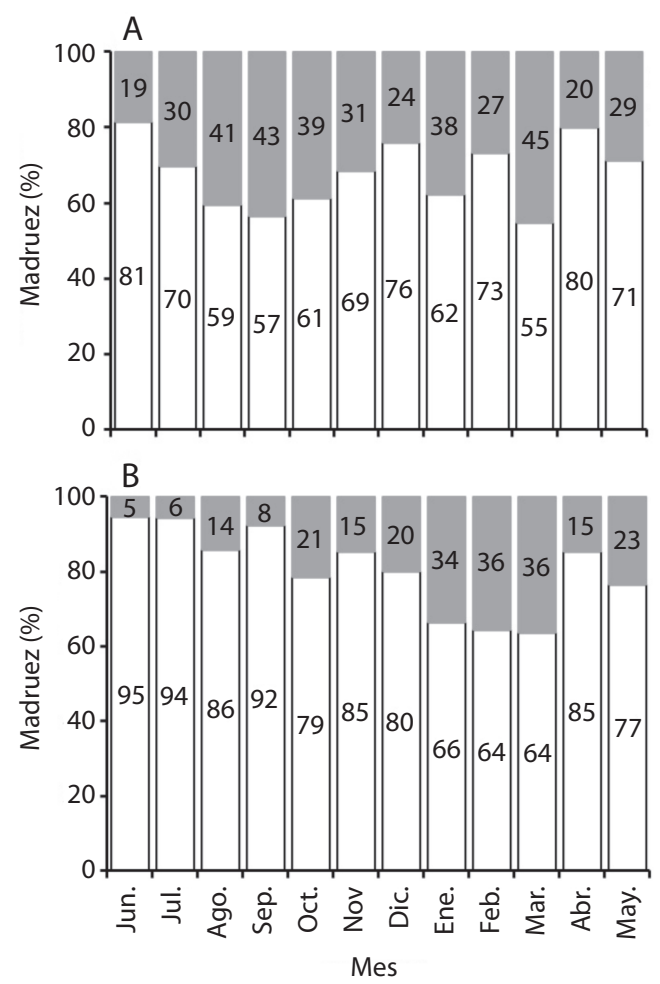

Talla media de madurez: La talla media de madurez en las hembras fue de $129.34 \mathrm{~m}$ de LT con valores de los parámetros de la función logística $\mathrm{a}=6.86 \mathrm{y} \mathrm{b}=-0.05$, con un $\mathrm{r} 2$ de 0.98 (Fig. 5A). En los machos la talla media de madurez se estimó en $97.77 \mathrm{~mm}$ de $\mathrm{LT}(\mathrm{a}=9.66$ y $b=-0.10)$, con un $r 2$ de 0.92 (Fig. 5B). Al comparar la talla media de madurez en hembras y machos con su respectiva talla media de captura (Fig. 3), se encontraron diferencias estadísticamente significativas (Hembras: Chi2 $=15$ 223.23; $\mathrm{p}=0.000$; machos: Chi2 $=17$ 988.74; $\mathrm{p}=0.000$ ).

Fig. 4. Estadios de madurez para (A) hembras y (B) machos de Farfantepenaeus notialis, obtenidos durante los muestreos desde Junio 2012 hasta Mayo 2013. Inmaduros: gris, Maduros: blanco.

Fig. 4. Stages of maturity to (A) females and (B) males of Farfantepenaeus notialis, obtained during the samplings from June of 2012 to May of 2013. Immature: grey, Mature: white. 

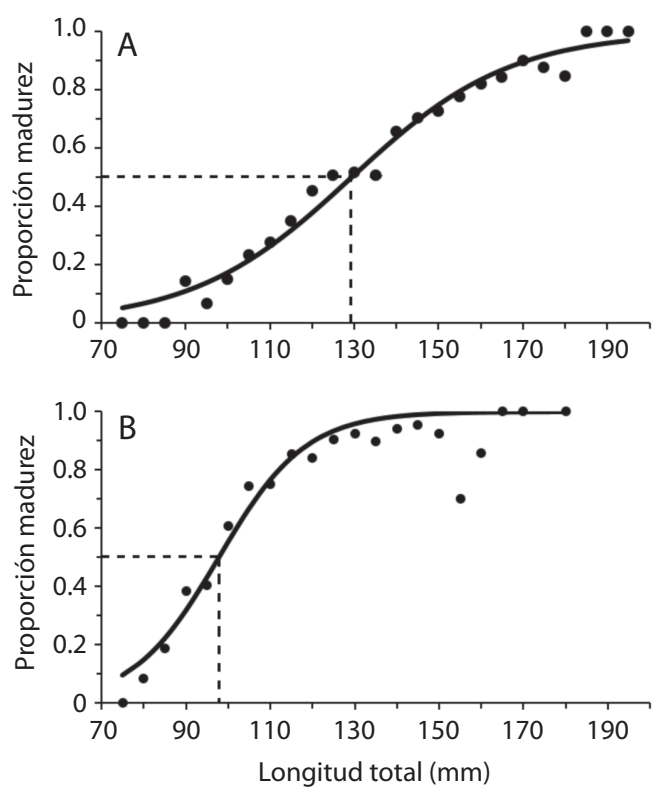

Fig. 5. Talla media de madurez $\left(\mathrm{LT}_{50 \%}\right)$ para (A) hembras y (B) machos de Farfantepenaeus notialis, obtenida de los datos de los muestreos desde junio 2012 hasta mayo 2013. Fig. 5. Mean size at sexual maturity $\left(\mathrm{LT}_{50 \%}\right)$ to $(\mathrm{A})$ females and (B) males of Farfantepenaeus notialis, obtained of the sampling data from June of 2012 to May of 2013.

\section{DISCUSIÓN}

La población de camarón rosado ( $F$. notialis) en el Caribe colombiano se caracterizó por tener un gran porcentaje de hembras maduras en todo el año $(>50 \%)$, indicando que la reproducción es continua durante todo el año pero con dos picos de máximo desove, uno entre octubre y diciembre y el otro entre abril y junio. Esto coincide con lo encontrado para los camarones peneidos que comúnmente muestran dos picos de desove por año (García \& Le Reste, 1986; Pauly et al., 1984; García, 1985). $\mathrm{La} \mathrm{LT}_{50 \%}$ en este estudio fue de $129.34 \mathrm{~mm}$ de LT para las hembras y $97.77 \mathrm{~mm}$ de LT para los machos, la cual es similar para las hembras en estudios previos $\left(\mathrm{LT}_{50 \%}=136.7 \mathrm{~mm}\right.$ ) (Paramo \& Saint-Paul, 2010). Además, la talla media de captura (TMC) siempre fue mayor que la $\mathrm{LT}_{50 \%}$ para ambos sexos. Debido a que el desove es la base de la renovación y conservación del stock de un recurso pesquero, estudios sobre la reproducción de los peneidos explotados son críticos para el desarrollo de estrategias de manejo (Aragón-Noriega \& Alcántara-Razo, 2005). Una medida central para el manejo es proteger el periodo y las áreas reproductivas (García, 1985). Adicionalmente, se ha sugerido que las estrategias de manejo deben permitir a las hembras reproducirse al menos una vez antes de ser capturadas (Aragón-Noriega \& Alcántara-Razo, 2005). Por lo tanto, las medidas de manejo que se deben adoptar en pesquerías de crustáceos para asegurar una explotación eficiente y sostenible, incluyen restricciones de captura y esfuerzo, talla mínima legal de desembarque (superior a la $\mathrm{LT}_{50 \%}$ ), estaciones cerradas (vedas) para proteger la reproducción y el reclutamiento y áreas protegidas. En este sentido, uno de los principales elementos de presión para el stock es la alta tasa de explotación en el periodo reproductivo, entonces es probable que esto pueda conllevar a riesgo de sobreexplotación o fracaso en el reclutamiento en el medio y largo plazo (Lizárraga-Cubedo et al., 2008). No obstante, también se ha demostrado en camarones peneidos que un esfuerzo pesquero alto en las etapas de juveniles en áreas de crianza y durante el reclutamiento puede conllevar a una sobreexplotación (Gracia, 1995, 1997, 2004). Por lo tanto, se deben diseñar estrategias de manejo para proteger los juveniles (Pérez-Castañeda \& Defeo, 2000; May-Kú \& Ordóñez-López, 2006) Además, debido a su ciclo de vida corto ( 2 años) (García, 1996; Leal-Gaxiola, López-Martínez, Chávez, Hernández-Vásquez \& Méndez-Tenorio, 2001; Charnov \& Hannah, 2002; López-Martínez, Arreguín-Sánchez, Hernández-Vásquez, García-Juarez \& Valenzuela-Quiñonez, 2003; López-Martínez et al., 2005), características de alto potencial reproductivo tales como fecundidad alta, generaciones anuales, maduración temprana y estrategia oportunista para aprovechar condiciones ambientales favorables, los camarones peneidos pueden recuperarse en un relativo corto plazo después de una sobreexplotación (Gracia, 1991, 1996). En este sentido, debido a que la pesquería de camarón de aguas someras 
en el Caribe colombiano llegó a tener unas 120 embarcaciones en la década de los 90's (Barreto \& Borda, 2008; Paramo \& Saint-Paul, 2010), pero actualmente y desde hace unos 10 años el esfuerzo pesquero disminuyó a unas ocho embarcaciones, se podría suponer que la pesquería de camarón de aguas someras este en una fase de recuperación o que probablemente pueda estar recuperada. No obstante, debido a que no existe temporada de veda en la pesquería de CAS en el Caribe colombiano, se recomienda una veda ya sea en la época reproductiva (noviembre-diciembre) o en la época de reclutamiento (marzo) o una combinación de las dos con periodo de veda en diciembre (reproducción) y otro periodo en marzo (reclutamiento). Por último, algo muy importante es no aumentar el esfuerzo pesquero hasta que se realice un monitoreo pesquero y conocer la real recuperación de la población de CAS en el Caribe colombiano.

\section{AGRADECIMIENTOS}

Este trabajo es una contribución del grupo de investigación Ciencia y Tecnología Tropical (CITEPT) Universidad del Magdalena (Colombia), con el apoyo científico del LeibnizZentrum für Marine Tropenökologie (ZMT), Alemania. Agradecemos a los Capitanes del Barco "Adriatic" David Guillem y Alfonso Guillem y a su tripulación por su interés y apoyo para que se realizara la investigación. Agradecemos a los estudiantes del Programa de Ingeniería Pesquera de la Universidad del Magdalena del grupo de investigación CITEPT quienes participaron en los muestreos a bordo de la embarcación y análisis en laboratorio. El trabajo fue financiado por el Instituto colombiano de Desarrollo Rural (INCODER), la Autoridad Nacional de Pesca y Acuicultura AUNAP y la Universidad del Magdalena, convenio 790-2011 (INCODER/Unimagdalena), proyecto "Dinámica y estructura poblacional de las especies de camarón de aguas someras y profundas del Caribe colombiano, con fines de ordenación y resolución de conflictos", y convenio 018-2012 (AUNAP/Unimagdalena) proyecto "Evaluación directa de la Biomasa y distribución espacial de las especies de las especies de Camarón de Aguas someras (CAS) en el Caribe colombiano".

\section{RESUMEN}

El camarón de aguas someras, es uno de los recursos de mayor importancia socioeconómica en la región Caribe. La falta de información biológica pesquera, es una preocupación de los administradores pesqueros. Por tanto, el presente estudio tiene como objetivo conocer la época reproductiva, la estructura de tallas y la talla media de madurez del camarón de aguas someras (Farfantepenaeus notialis) como insumo para la ordenación y manejo pesquero en el Caribe colombiano. El estudio se llevó a cabo desde junio del 2012 hasta mayo del 2013 en el Caribe colombiano. Se recolectaron 5356 individuos, los cuales fueron identificados, clasificados y conservados para su análisis en el laboratorio, donde se analizaron las muestras tomando datos de talla, peso, sexo y estadio gonadal. Se encontraron diferencias significativas en la razón de sexo durante todos los meses muestreados, con una mayor proporción de hembras. Se presentaron hembras maduras durante todo el año, pero con dos picos reproductivos de máximo desove, uno entre octubre y diciembre y el otro entre abril y junio. La talla media de captura (TMC) durante todo el muestreo para las hembras fue de $148.0 \mathrm{~mm}$ de longitud total (LT) y para los machos fue de $122.5 \mathrm{~mm}$ LT. La talla media de madurez $\left(\mathrm{LT}_{50 \%}\right)$ para las hembras y machos fue de $129.34 \mathrm{~mm}$ LT y $97.77 \mathrm{~mm}$ LT, respectivamente. La TMC siempre fue mayor que la $\mathrm{LT}_{50 \%}$ para ambos sexos. Debido a la gran disminución del esfuerzo pesquero en el Caribe colombiano durante los últimos 10 años, se espera que este recurso se encuentre en una fase de recuperación o ya esté recuperado.

Palabras clave: camarón, Farfantepenaeus notialis, reproducción, talla media de madurez, Caribe colombiano.

\section{REFERENCIAS}

Aragón-Noriega, E. A. \& Alcántara-Razo, E. (2005). Influence of sea surface temperature on reproductive period and size at maturity of brown shrimp (Farfantepenaeus californiensis) in the Gulf of California. Marine Biology, 146, 373-379.

Barreto, C. \& Borda, C. (2008). Evaluación de recursos pesqueros colombianos. Bogotá: ICA Produmedios.

Belcari, P., Viva, C., Mori, M., \& De Ranieri, S. (2003). Fishery and biology of Aristaeomorpha foliacea (Risso, 1827) (Crustacea: Decapoda) in the Northern Tyrrenien Sea (Western Mediterranean). Journal of Northwest Atlantic Fishery Science, 31, 195-204. 
Carlucci, R., D’Onghia, G., Sion, L., Maiorano, P., \& Tursi, A. (2006). Selectivity parameters and size at first maturity in deep-water shrimps, Aristaeomorpha foliacea (Risso, 1827) and Aristeus antennatus (Risso, 1816), from the north-western Ionian Sea (Mediterranean Sea). Hydrobiologia, 557, 145-154.

Charnov, E. L. \& Hannah, R. W. (2002). Shrimp adjust their sex ratio to fluctuating age distributions. Evolutionary Ecology Research, 4, 239-246.

Dall, W., Hill, B. J., Rothlisberg, P. C., \& Staples, D. J. (1990). The biology of the Penaeidae. Advances in Marine Biology, 27, 1-489.

Desantis, S., Labate, M., Maioramo, P., Tursi, A., Labate, G. M., \& Ciccarelli, M. (2001). A histochemical and ultrastructural study of oogenesis in Aristaeomorpha foliacea (Risso, 1827). Hydrobiologia, 449, 253-259.

Echeverría-Sáenz, S. \& Wehrtmann, I. S. (2011). Egg production of the commercially exploited deepwater shrimp, Heterocarpus vicarius (Decapoda: Pandalidae), Pacific Costa Rica, Central America. Journal of Crustacean Biology, 31(3), 434-440.

FAO. (1995). Código de conducta para la pesca responsable. Roma, FAO.

Fischer, W., Bianchi, G., \& Scott, W. B. (Eds.). (1981). FAO Species identification Sheets for Fishery Purposes. Volumen VII. Eastern Central Atlantic; fishing area 34 and part of 47. Roma: Canada Funds-in-Trust \& FAO.

Fischer, W., Krupp, F., Schneider, W., Sommer, C., Carpenter, K. E., \& Niem, V. H. (1995). Guía FAO para la identificación de especies para los fines de la pesca. Pacifico centro-oriental. Volumen II. Vertebrados Parte 1. Roma: FAO.

García, S. \& Reste, L. L. (1986). Ciclos vitales, dinámica, explotación y ordenación de las poblaciones de camarones peneidos costeros. Roma: FAO Doc. Téc. Pesca.

García, S. (1985). Reproduction, stock assessment models and population parameters in exploited penaeid shrimp populations. In P. C. Rothlisberg, B. J. Hill, \& D. J. Staples (Eds.), Second Australian National Prawn Seminar, NPS2 (pp. 139-158). Cleveland.

García, S. (1989). The management of coastal penaeid shrimp fisheries. In J. F. Caddy (Ed.), Marine Invertebrate Fisheries: Their Assessment and Management (pp. 281-306). New York: Wiley.

García, S. (1996). The precautionary approach to fisheries and its implications for fishery research, technology and management: an updated review. In Precautionary Approach to Fisheries. Part 2: Scientific papers (pp. 1-75). Roma: FAO.

Gotelli, N. J. \& Ellison, A. M. (2004). A primer of Ecological Statistics. Sunderland: Sinauer Associates, Inc.
Gracia, A. (1991). Spawning stock-recruitment relationships of white shrimp in the Southwestern Gulf of Mexico. Transactions of the American Fisheries Society, 120, 519-527.

Gracia, A. (1995). Impacto de la pesca artesanal sobre la producción de camarón rosado Penaeus Farfantepenaeus duorarum Burkenroad, 1939. Ciencias Marinas, 21, 343-359.

Gracia, A. (1996). White shrimp Penaeus setiferus recruitment overfishing. Marine and Freshwater Research, 47, 59-63.

Gracia, A. (1997). Simulated and actual effects of the brown shrimp, Penaeus aztecus, closure in Mexico. Marine Fisheries Review, 59, 18-24.

Gracia, A. (2004). Aprovechamiento y conservación del recurso camarón. In M. Caso, I. Pisanty, \& E. Ezcurra (Eds.), Diagnóstico ambiental del Golfo de México (Vol. 2, pp. 713-725). Secretaría de Medio Ambiente y Recursos Naturales, Instituto Nacional de Ecología, A. C., Harte Research Institute for the Gulf of México Studies.

Guillet, R. (2008). Global study of shrimp fisheries. Roma: FAO Fisheries Technical Papers. No. 475.

Kapiris, K. \& Thessalou-Legaki, M. (2006). Comparative fecundity and oocyte size of Aristaeomorpha foliacea and Aristeus antennatus in the Hellenic Ionian Sea (E. Mediterranean) (Decapoda: Aristeidae). Acta Zoologica, 87, 239-245.

Kapiris, K. \& Thessalou-Legaki, M. (2009). Comparative Reproduction Aspects of the Deep-water Shrimps Aristaeomorpha foliacea and Aristeus antennatus (Decapoda, Aristeidae) in the Greek Ionian Sea (Eastern Mediterranean). International Journal of Zoology, doi:10.1155/2009/979512 Article ID 979512, 9 p.

Leal-Gaxiola, A., López-Martínez, J., Chávez, E. A., Hernández-Vásquez, S., \& Méndez-Tenorio, F. (2001). Interannual variability of the reproductive period of the brown shrimp, Farfantepenaeus californiensis (Holmes, 1900) (Decapoda, Natantia). Crustaceana, 74, 839-851.

Lee, S. Y. (1999). Tropical mangrove ecology: physical and biotic factors influencing ecosystem structure and function. Australian Journal of Ecology, 24, 355-366.

Lizárraga-Cubedo, H. A., Pierce, G. J., \& Santos, M. B. (2008). Reproduction of crustaceans in relation to fisheries. In E. Mente (Ed.), Reproductive biology of crustaceans, case studies of decapod crustaceans (pp. 169-222). Enfield: Science Publishers.

Loneragan, N. R. \& Bunn, S. E. (1999). River flows and estuarine ecosystems: implications for coastal fisheries from a review and a case study of the Logan River, South-East Queensland. Australian Journal of Ecology, 24, 431-440. 
López-Martínez, J., Arreguín-Sánchez, F., HernándezVásquez, S., García-Juarez, A. R., \& ValenzuelaQuiñonez, W. (2003). Interanual variation of growth of the brown shrimp Farfantepenaeus californiensis and its relationship to temperature. Fisheries Research, 61, 95-105.

López-Martínez, J., Rábago-Quiroz, C., Nevárez-Martínez, M. O., García-Juárez, A. R., Rivera-Parra, G., \& Chávez-Villalba, J. (2005). Growth, reproduction, and size at first maturity of blue shrimp, Litopenaeus stylirostris (Stimpson, 1874) along the east coast of the Gulf of California, Mexico. Fisheries Research, 71(1), 93-102.

May-Kú, M. A. \& Ordóñez-López, U. (2006). Spatial patterns of density and size structure of penaeid shrimps Farfantepenaeus brasiliensis and Farfantepenaeus notialis in a hypersaline lagoon in the Yucatán Peninsula, Mexico. Bulletin of Marine Science, 79(2), 259-271.

Minello, T. J. \& Zimmerman, R. J. (1991). The role of estuarine habitats in regulating growth and survival of juvenile penaeid shrimp. In P. F. Deloach, W. J. Dougherty, \& M. A. Davidson (Eds.), Frontiers of shrimp research. Developments in Aquaculture and
Fisheries Science (Vol. 22, pp. 1-16). Amsterdam: Elsevier Science.

Paramo, J. \& Saint-Paul, U. (2010). Morphological differentiation of southern pink shrimp Farfantepenaeus notialis in Colombian Caribbean Sea. Aquatic Living Resources, 23(1), 95-101.

Pauly, D., Ingles, J., \& Neal, R. (1984). Application to shrimp stocks of objective methods for the estimation of growth, mortality and recruitment-related parameters from length-frequency data (ELEFAN I and II). In J. A. Gulland \& B. J. Rothschild (Eds.), Penaeid shrimps. Their biology and management (pp. 220234). Fishing New Books.

Pérez-Castañeda, R. \& Defeo, O. (2000). Population structure of penaeid shrimp Farfantepenaeus notialis in its new range extension for the Gulf of Mexico. Bulletin of Marine Science, 67(3), 1069-1074.

Tavares, M. (2002). Shrimps. In K. E. Carpenter (Ed.), The living marine resources of the Western Central Atlantic. Introduction, molluscs, crustaceans, hagfishes, sharks, batoid fishes, and chimaeras. FAO Species Identification Guide for Fishery Purposes and American Society of Ichthyologists and Herpetologists Special Publication No. 5 (Vol. 1, pp. 251-291). Roma: FAO. 
